\title{
Ballistic Transport in Semiconductor Quantum Wires in the Presence of Defects
}

\author{
A. R. Rocha and J. A. Brum \\ Laboratório Nacional de Luz Síncrotron, ABTLuS, 13083-970, Campinas, SP, Brazil and \\ DFMC-Instituto de Física Gleb Wataghin,Unicamp,13.081-970 Campinas, SP, Brazil
}

Received on April 27, 2001

\begin{abstract}
We present calculations of the conductance in semiconductor quasi-one-dimensional systems using the Landauer formalism. We consider the effects on the transport properties inside semiconductor quantum waveguides of different shapes when a defect is located either in the wire region or in the quasi-two-dimensional region. We observe changes on the plateau's threshold when the defect is placed inside the wire and lowering of the conductance plateaus themselves below the conductance quantum $G_{0}=2 \mathrm{e}^{2} / \mathrm{h}$ when the defect is outside the wire.
\end{abstract}

\section{Introduction}

The first experimental evidences of conductance quantization through one-dimensional (1D) quantum systems were made by van Wees et al. [1] and Wharam et al. [2], both in 1988[3]. These experiments were conducted on quantum point contacts (QPC) made from doped semiconductor heterostructures which form a quasi-two-dimensional electron gas (2DEG). The system is in the Electronic Quantum Limit and from now on we assume it to be a pure $2 \mathrm{DEG}$ that is, we neglect the scattering with the higher heterostructure levels. The electron flow through the point contact is constrained to $1 \mathrm{D}$ by by means of a negative potential bias applied on the metal plates of the contact.

The fabrication of these structures is made by epitaxial growth. In the case of this study, we considered GaAs-AlGaAs heterostructures with a layer of modulated n-doped AlGaAs. In general some of the Alu- minum atoms will defuse through the interface of the junction acting as scattering centers for the electrons.

The first theoretical considerations concerning defects on QPC's were made by Nixon et al.[4], who considered random distribution of defects along the structure. Recently, Topinka et al.[5], recorded electron flow through a QPC under the influence of an AFM tip, placed outside the point contact and functioning as a probe for the electron wavefunction.

Our aim was to study theoretically the effects that the existence of these scattering centres might have on the conductance plateaus on two different shapes of quantum waveguides. The first one being a QPC (one constriction) and the second one an open quantum dot (OQD) structure (two narrow constrictions and one wide constriction)(see Fig.1).

\section{Theoretical Modelling}

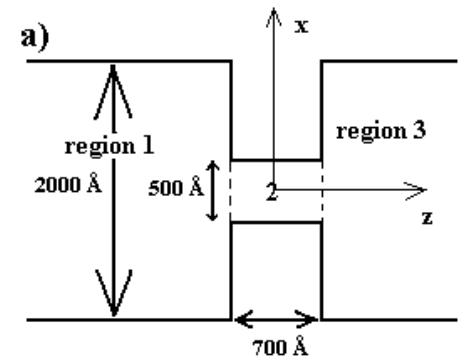

b)

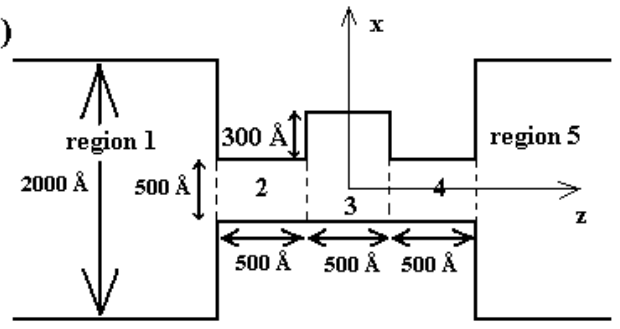

Figure 1. Schematic illustration of the the two structures that were studied and their confining potential 1a) QPC 1b) OQD.

In order to simulate the two structures which were considered we used the voltage profile schematized on Fig. 1a. and 1b. within the envelope function and ef- fective mass approximations [6]. The eigenstates of the continuum of the electron bath were considered to be discretized by the solutions of a quantum well with infi- 
nite potential barriers and length much greater than the dimension of the constriction. The constricted regions were depicted as square wells with constant potential barrier $V_{0}$.

The solutions inside the constricted regions were found by projecting the basis of the electron bath $\left(\mid n_{x}>\right)$ onto the Hamiltonian of the system. Therefore, we write the wavefunctions:

$$
\left|\psi_{m}^{\{I\}}>=\sum_{n}^{N} a_{m n}\right| n_{x}>\mid 1_{z}>
$$

the $a_{m n}$ 's are determined by projecting the Hamiltonian

$$
H_{\text {narrow }}=T_{e}+V_{0} Y\left[|x|-\frac{L_{x}}{2}\right]
$$

for the narrow constrictions on Fig. 1a. and 1b. and

$$
H_{w i d e}=T_{e}+V_{0} Y\left[x+\frac{L_{x}}{2}\right]+V_{0} Y\left[x-\left(L_{x} 2+L w_{x}\right)\right]
$$

for the wide constriction on Fig. 1b into Eq. 1 and diagonalizing the eigenvalue problem.

The defect is simulated by a positive square barrier of height $V_{\text {def }}$ placed at different regions of the waveguide. The contribution to the Hamiltonian is $\quad V_{d e f}\left(Y\left[x-\left(x_{D}-\frac{L_{D}}{2}\right)\right]-Y\left[x-\left(x_{D}+\frac{L_{D}}{2}\right)\right]\right)$, added to either the narrow or the wide constriction Hamiltonian depending on the position of the defect.

The electrons were considered to be injected with an energy $E$, which must be conserved along the structure. Therefore, the wavevector for each region is given by:

$$
k_{y i}^{\{I\}}=\sqrt{\frac{2 m^{*}}{\hbar^{2}}\left(E-\epsilon_{i}^{I}\right)}
$$

where $\epsilon_{i}^{I}$ is the energy of the i-th level of the well in $\mathrm{x}$-direction for the I-th region.

Hence, the total wavefuntion can be writen, for each region:

$$
\begin{array}{r}
\left|\Psi>_{n^{\prime}}^{1}=e^{i k_{n^{\prime}}^{1} y}\right| n_{x}^{\prime}>+\sum_{n}^{N} r_{n^{\prime} n} e^{-i k_{n}^{1} y} \mid n_{x}> \\
\left|\Psi>_{n^{\prime}}^{\{I\}}=\sum_{j}^{N}\left(\alpha_{n^{\prime} j} e^{i k_{j}^{\{I\}}}+\beta_{n^{\prime} j} e^{-i k_{j}^{\{I\}}}\right)\right| \psi_{j}^{\{I\}}> \\
\left|\Psi>_{n^{\prime}}^{3}=\sum_{n}^{N} t_{n^{\prime} n} e^{i k_{n}^{3} y}\right| n_{x}>
\end{array}
$$

By imposing the condition of continuity of both the wavefunction and the flux at each interface, we obtain a system of non-homogeneous linear equations that can be solved for the r's and t's (the reflection and transmission coefficients). The conductance can then be calculated by the Landauer formula[7]:

$$
G(E)=\sum_{n^{\prime}} \sum_{i}\left|\frac{k_{i}^{3}}{k_{n^{\prime}}^{1}}\right|\left|t_{i}\right|^{2}
$$

This Procedure is know as Mode Matching and has been widely used for this kind of problem $[8,9]$.

\section{Results and Discussion}

On our calculations we considered a square defect $10 \AA \times 10 \AA$ and $600 \mathrm{meV}$ high (the same potencial estimated for the depleted region that define the structure). This is higher than a structural defect for which $\mathrm{V}_{\text {def }} \backsim 300 \mathrm{meV}$, but simulates a depleted area induced by an electrostatic contact. The effective mass was considered to be $0.067 m_{0}$. For the case with one constriction, the contact was taken to be $500 \AA\left(L_{x}\right)$ wide $700 \AA$ long. For the case of two constrictions the wire is as wide as the first one and each constriction is $500 \AA$ long.

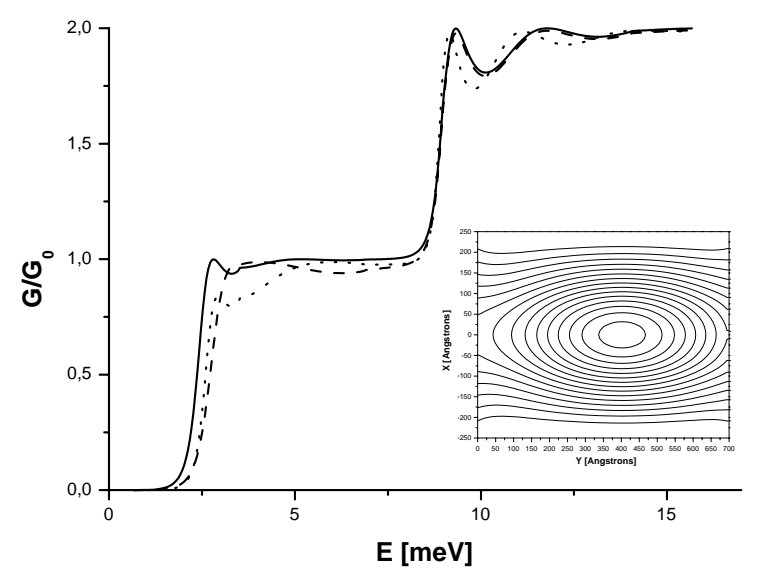

Figure 2. a) Conductance as a function of initial electron energy with the defect placed on $\mathrm{x}=0 \AA$. $\mathrm{b}$ ) Projection on the xy plane of the Electron Density inside the Point Contact for an initial energy of $3 \mathrm{meV}$.

The conductance curves shown on Fig. 2 were made by placing the defect inside the wire on two different places along the $y$-axis for the QPC case. We can observe that the defect shifts the $1^{\text {st }}$ plateau threshold to higher energies. We can also see that the second plateau remains unchanged.

On Fig. 3 the defect is kept at $x=130 \AA$ And is made to vary in the $y$ direction. It is evident that the opposite behavior of Fig. 2 now occurs.

The interpretation of this phenomenon relies on the electron densities depicted as density plots on Fig. 2 and 3. The low energy wavefunction (first conduction mode) presents a high electron density closer to the $y$ axis, so the effect of the defect on the electrons will be higher when $x \sim 0$. On the other hand, when the energy is $11 \mathrm{meV}$, the wavefunction has a knot and the charge density is concentrated parallel to the $y$-axis. In that case the electrons are not influenced by the defect, when the latter is placed at $x=0$. 


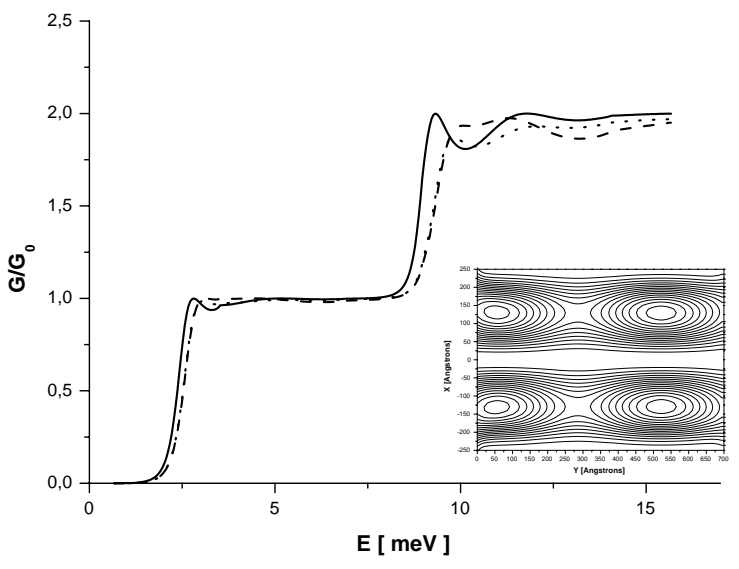

Figure 3. a) Conductance as a function of initial electron energy with the defect placed on $x=130 \AA$. b) Projection on the xy-plane of the Electron Density inside Point Contact for an initial energy of $11 \mathrm{meV}$.

The opposite happens for the second plateau, when we have a combined effect of the two first eigenstates of the constriction. In this case, the defect displaced from the wire centre shows a stronger influence on the second plateau as a consequence of the second eigenstate wavefunction spatial distribution.

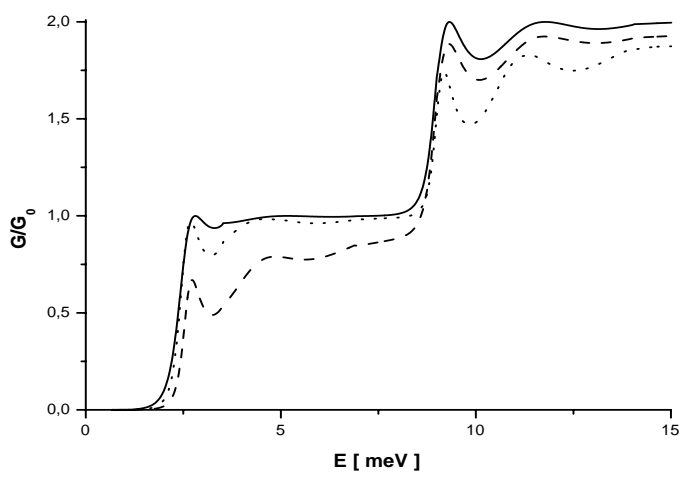

Fig. 4. Conductance as a function of initial electron energy with the defect placed outside the wire (at $\mathrm{y}=800 \AA$ ).

When we place the defect outside the QPC, as seen in Fig. 4, there is no energy threshold shift. However, the conductance plateaus appear below $\mathrm{G}_{0}$ due to the scattering of the electrons by the defect, preventing some of them to reach the drain. The influence of the defect's position has the same electron density interpretation that was given to the phenomena in Fig. 2 and 3.

From the analysis of the conductance curves for the OQD we can point out in Fig. 5 the bound state that names this structure[10]. The state signature is a peak on the conductance due to resonant tunnelling of the electrons through the quantum dot ground state. The greater confinement on the $\mathrm{x}$ direction in the narrow constriction (outside the dot) gives rise to a virtual confining potential in the y direction on both sides of the dot (wider constriction).

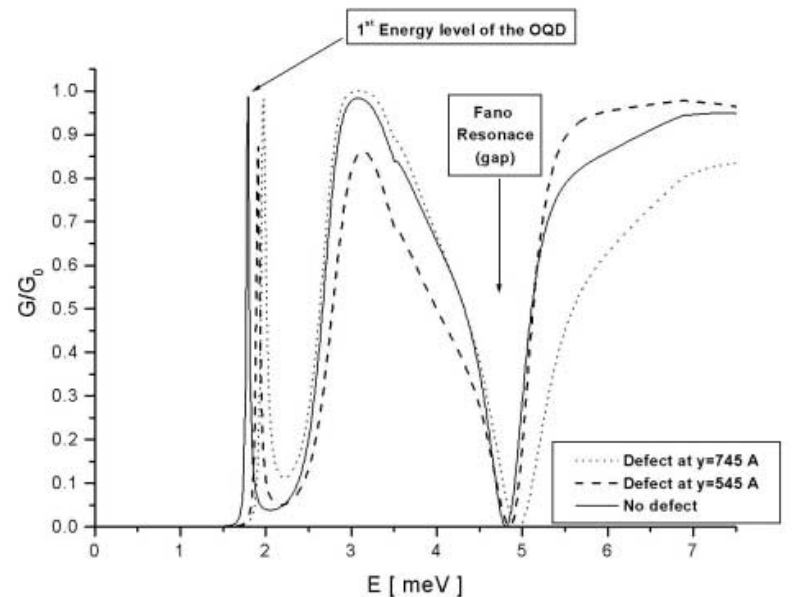

Figure 5. Conductance as a function of initial electron energy with the defect placed at $\mathrm{x}=0$ Affor the for the OQD case.

We can also see a Fano-resonance-type curve which is due to the repulsion of the second energy level of the OQD and the first band of the conducting channel. Hence, we have the formation of a band gap in the conductance showing the destructive interference between the channels.

By placing the defect inside the dot there is a raise in the energy along the $\mathrm{x}$ direction, thus blue-shifting the bound state and the Fano-resonance. The conductance plateau a slight decrease. Relying on what was said about Fig. 4, we can imagine that the defect acts somehow as if it were on the 2DEG and the lowering of the conductance plateaus is due to the scattering on the defect and on the first two interfaces only.

In summary, we have seen that the existence of defects has an important role on ballistic transport. In general, the defects will appear in far greater number and randomly distributed throughout the structure and their main effect will be to lower the conductance plateaus.

\section{Acknowledgments}

We are grateful to $\mathrm{CNPq}$ and (Brazil) Fapesp (Brazil) for the financial support.

\section{References}

[1] B.J. van Hees, H. van Houten, C.W.J. Beenaker, J.G. Willianson, L.P. Kouwenhoven, D. van der Marel, and C. T. Foxon, Phys. Rev. Lett. 60, 848 (1988).

[2] D.A. Wharam, T.J. Thornton, R. Newbury, M. Pepper, H. Ahmed, J.E.F. Frost, D.G. Hasko, D.C. Peacock, D.A. Ritchie, and G.A.C. Jones, J. Phys. C 21, L209 (1988).

[3] for a review see W.J. Beenakker and H. van Houten, (1991). 
[4] J.A. Nixon, J.H. Davies, and H.U. Baranger, Supperlattices and Microstructures, 9, 187 (1991).

[5] M.A. Topinka, B.J. LeRoy, S.E.J. Shaw, E.J. Heller, R.M. Westervelt, K.D. Maranowski, and A. C. Gossard, Science, 289, September (2000).

[6] G. Bastard, Wave Machanics Applied to Semiconductor Heterostructures, Les Editions de Physique, Les Ulis, (1988).

[7] M. Bütikker, Y. Imry, R. Landauer, and S. Pinhas, Phys.
Rev. B, 31, 6207 (1985).

[8] G. Bastard, J. A. Brum, and R. Ferreira, Solid State Physics, 44, 229 (1991).

[9] D.K. Ferry and S. M. Goodnick, Transport in Nanostructures, Cambridge Studies in Semiconductor Physics and Microelectronics, Cambridge University Press, Cambridge, 1997.

[10] J.A. Brum, Physical Review B 43, 12082, (1991). 